\title{
Historische Landnutzung und Siedlungsentwicklung in Flussauen und Hochwasserschutz: Das Beispiel der Traisen und St. Pöltens 1870-2000
}

\author{
Gertrud Haidvogl · Jürgen Eberstaller · Doris Eberstaller-Fleischanderl · Bernhard Fraiß · Hannes Gabriel · \\ Severin Hohensinner
}

Online publiziert: 7. März 2018

(c) Der/die Autor(en) 2018. Dieser Artikel ist eine Open-Access-Publikation.

Zusammenfassung Auen sind ein wichtiger Teil des Ökosystems Flusslandschaft. Ihre Ausdehnung und ökologische Funktionsfähigkeit haben vor allem in den letzten 200 Jahren durch die zunehmende menschliche Nutzung stark abgenommen. Die Kolonisierung von Flussauen ging Hand in Hand mit einem grundlegenden Wandel im Hochwasserschutz. Dieser Artikel beschreibt anhand des Beispiels der Traisenflusslandschaft im Bereich der Stadt St. Pölten die parallel verlaufenden und sich gegenseitig beeinflussenden Prozesse von Landnutzungsänderung, Siedlungsentwicklung und Ausbau technischer Hochwasserschutzmaßnahmen. Im räumlichen und zeitlichen Verlauf der Siedlungsentwicklung St. Pöltens in den Traisenauen waren Industrialisierung, Urbanisierung, städtische Planung und technische Hochwasserschutzpraktiken treibende Faktoren. Die Stadt dehnte

\section{Mag. Dr. G. Haidvogl ( $\square)$. \\ DI Dr. S. Hohensinner \\ Institut für Hydrobiologie \\ und Gewässermanagement, \\ Universität für Bodenkultur Wien, \\ Gregor-Mendel-Straße 33, 1180 Wien, Österreich \\ gertrud.haidvogl@boku.ac.at}

DI Dr. J. Eberstaller .

DI D. Eberstaller-Fleischanderl

ezb - TB Eberstaller GmbH,

Schopenhauerstraße

82/12, 1180 Wien, Österreich

\section{B. Fraiß}

Ingenieurbüro für Kulturtechnik und Wasserwirtschaft, Zum

Holzapfel 15, 8664 Veitsch-St. Barbara, Österreich

\section{H. Gabriel}

DonauConsult Ingenieurbüro $\mathrm{GmbH}$, Klopstockgasse 34, 1170 Wien, Österreich sich im 20. Jahrhundert kontinuierlich in die Traisenauen aus. Diese Expansion beschränkte sich zunächst auf die Ränder des $\mathrm{HQ}_{100}$-Abflussraums, während entlang der seit 1909 existierenden Hochwasserschutzdämme bis 1960 ein Grünland- bzw. Auwaldstreifen verblieb. Dieser wurde bewusst von Bebauung freigehalten und bei größeren Hochwässern überschwemmt. Nach dem Hochwasser von 1959 gab die Stadt auch die traisennahen Flächen als Bauzonen frei. Diese Entscheidung fiel parallel zum Entschluss, den Hochwasserschutz auf ein 100-jährliches Abflussereignis auszubauen. In den folgenden Jahrzehnten entstanden auch direkt an der Traisen dauerhafte Wohngebäude und -siedlungen. Aktuell stellen die vorhandenen Nutzungen sowie die dadurch verursachten ökologischen Folgen das Gewässermanagement vor große Herausforderungen. Diese können nur mithilfe von einzugsgebietsbezogener Planung, der Integration von vielfältigen Anforderungen inklusive der Ökologie und der Berücksichtigung der Raumplanung als Steuerungsinstrument gelöst werden.

Historic land use and settlement development in river floodplains and flood protection: the example of Traisen and St. Pölten 1870-2000

Abstract Floodplains are an impor-
tant part of riverine landscapes. Their
expansion and ecological functioning
have declined sharply, especially in the
last 200 years due to increasing human
use. The colonization of floodplains
went hand in hand with a fundamental
change in flood protection practices.
Using the example of the Traisen river
landscape in the area of the city of
St. Pölten, this article describes the
parallel and mutually influencing pro-
cesses of land use change, settlement development and the evolution of technical flood protection measures. In the spatial and temporal patterns of St. Pölten's settlement development in the Traisen floodplains industrialization, urbanization, urban planning and technical flood protection practices were driving factors. In the 20th century, the city expanded continuously towards and in the river floodplains. This expansion was initially limited to the edges of the 100-year flood area, while along the flood protection dikes, which were erected between 1905 and 1909 a grassland and floodplain forest section remained until 1960. This zone was deliberately kept free of buildings as it was inundated during major floods. After the flood of 1959, the city also designated the sections close to the river as construction zones. Simultaneously, the decision was taken to extend flood protection to a 100-year runoff event. In the following decades, permanent residential buildings and settlements were built directly along the Traisen River. Currently, the existing floodplain uses and the resulting ecological consequences pose major challenges for water management. These can only be addressed based on planning on catchment scale, integration of a variety of use requirements including ecology and the consideration of spatial planning as guiding principles.

\section{Einleitung}

Auen sind ein wichtiger Teil des Ökosystems Flusslandschaft. Durch intensive menschliche Nutzungen haben Ausmaß und ökologische Funktionsfähigkeit vor allem in den letzten 200 Jahren stark abgenommen. In Österreich sind aktuell 823 Auenstandorte mit einer Gesamtfläche von $955 \mathrm{~km}^{2}$ ausgewiesen (Hein et al. 2014). Im Jahr 2003 zeigte eine Studie, dass entlang der 50 größten österreichischen Flüsse (Einzugsgebiet 
größer als $500 \mathrm{~km}^{2}$ ) nur mehr $710 \mathrm{~km}^{2}$ von $4739 \mathrm{~km}^{2}$ oder $15 \%$ Auen im eigentlichen Sinn sind, das heißt, sie werden regelmäßig überflutet (Poppe et al. 2003; Muhar et al. 2004). Auf den verschwundenen Auenflächen wurden überwiegend Äcker (ca. 32\%) oder Grünland (ca. 27\%) kultiviert. Siedlungen und Industriestandorte nahmen $6 \%$ ein. Die Bedeutung von Flussauen als Wirtschafts- und Siedlungsräume liegt in Österreich wohl nicht zuletzt auch in der Topografie begründet. Von der gesamten Landesfläche (knapp $84.000 \mathrm{~km}^{2}$ ) sind nur etwa $37 \%$ $\left(31.100 \mathrm{~km}^{2}\right)$ als potenzieller Dauersiedlungsraum geeignet (Lexer und Linser 2005). Auen machten damit immerhin $15 \%$ dieses Dauersiedlungsraums aus.

Vor der Industrialisierung war die Jahrhunderte zurückreichende Nutzung von Flussauen (s. z. B. Haidvogl et al. 2011 zur Möll) oft an die Gewässerdynamik, an Überschwemmungsverhältnisse und andere Umweltfaktoren, wie z.B. Geländetopografie, angepasst. BewohnerInnen und NutzerInnen der Auen hatten viele Praktiken entwickelt, um bei Überflutungen die Schäden so weit wie möglich zu minimieren. So gab es z.B. im Erdgeschoss von Gebäuden keine fix verankerten Möbel oder Maschinen, damit man diese bei Überschwemmungen rasch in höher gelegene Stockwerke befördern konnte. In großen Städten, wie z.B. in Wien, wurden im Lauf der Zeit umfangreiche Notfallpläne entwickelt und formalisiert. Diese regelten in überfluteten Siedlungsräumen die Versorgung der betroffenen Menschen mit Wasser, Nahrung oder Holz, aber auch das Errichten von Notstegen, die Beobachtung des Abflusses oder Warnung und Entwarnung für Überflutungen (Haidvogl 2010; Haidvogl et al. 2013).

Die intensive Kolonisierung von Flussauen fand vor allem seit der zweiten Hälfte des 19. und im 20. Jahrhundert statt, gleichzeitig mit einem grundlegenden Wandel im Hochwasserschutz. Im Zeitalter der Industrialisierung unterstützte der Umstieg von Holz auf fossile Energieressourcen die Umsetzung von großräumigen, systematischen Flussregulierungen. Die Praxis des Hochwasserschutzes zielte nun nicht mehr darauf ab, die negativen Folgen von Überschwemmungen so weit wie möglich zu reduzieren, sondern vielmehr darauf, diese gänzlich $\mathrm{zu}$ verhindern. Die Besiedlung und Nutzung von Flussauen wurde zu einem scheinbar risikolosen Unternehmen. Das vermeintlich kontrollierbare und kontrollierte Hochwasserrisiko intensivierte die Nutzung der Auen. Tatsächlich wurde jedoch ein heute meist nicht mehr umkehrbarer und mit vielen unbeabsichtigten Nebenwirkungen verbundener Prozess in Gang gesetzt.

Am Ende des 19. Jahrhunderts erfasste die Kolonisierung von Flussauen zunächst vor allem städtische Räume. Ein eindrückliches Beispiel dafür ist die Entwicklung Wiens und die zunehmende Ausbreitung der Stadt in die Donauauen ab den 1850er-Jahren (Haidvogl et al. 2013). In den urbanen, aber auch in kleinstädtischen Regionen wurden die meist außerhalb der historischen Siedlungskerne liegenden Auen vor allem im 20. Jahrhundert zu begehrten, weil billigen Standorten für Industriebetriebe, Einkaufszentren oder hochrangige Verkehrsverbindungen. Zahlreiche Wohnsiedlungen und Gewerbezonen entstanden. Ab den 1970er-Jahren hatten Einfamilienhäuser immer öfter teure Heizungsanlagen in Kellern oder nicht mobile Einbauküchen in Erdgeschossen. Mit dem steigenden Schadenspotenzial mussten die Hochwasserschutzdämme oft höher ausgebaut werden. Aber auch die Nutzung der Auen für die Landwirtschaft wurde immer intensiver. Nach dem zweiten Weltkrieg führte der Flächenbedarf für eine angestrebte autarke Nahrungsproduktion zum Schlagwort des „zehnten Bundeslandes“, das an Gewässern und in Feuchtflächen durch Regulierung und Trockenlegung entstehen könnte (Ramsauer 1948). Unter diesem Titel wurden vor allem zwischen den 1950er- und 1970er-Jahren umfassende Entwässerungen und Gewässerregulierungen durchgeführt. So zeigte die Landnutzungsänderung in den Donauauen des östlichen Machlands in der zweiten Hälfte des 20. Jahrhunderts, dass mit der Einführung der Entschädigung von landwirtschaftlichen Hochwasserschäden ab 1975 immer mehr Äcker in überflutungsgefährdeten Zonen kultiviert wurden. Davor befanden sich diese außerhalb der Überschwemmungszone oder zumindest außerhalb der häufig erodierten, besonders dynamischen Flusszonen (Haidvogl 2008, 2010).

Heute befinden sich vor allem in urbanen Flussauen oft dicht besiedelte Siedlungsräume und Kulturgüter und dementsprechend große ökonomische Werte. Hochwasserschutz und Gewäs- sermanagement stellt dies nicht zuletzt vor dem Hintergrund des Klimawandels vor große Herausforderungen (Bundesministerium für Land- und Forstwirtschaft, Umwelt und Wasserwirtschaft 2005; Internationale Regierungskommission Alpenrhein \& Internationale Rheinregulierung 2005). Durch die Hochwasserschutzbauten und andere technische Maßnahmen zur Nutzung von Fließgewässern (Energiewirtschaft, Schifffahrt, Abwasserentsorgung) kam es $\mathrm{zu}$ ökologischen Veränderungen in einem zuvor nicht bekannten Ausmaß.

Dieser Artikel beschreibt anhand des Beispiels der Traisenflusslandschaft im Bereich der Stadt St. Pölten die parallel verlaufenden und sich gegenseitig beeinflussenden Prozesse von Landnutzungsänderung und Siedlungsentwicklung einerseits und den Ausbau der technischen Hochwasserschutzmaßnahmen andererseits. Für diesen in mehreren Phasen ablaufenden Prozess wurden folgende Fragen analysiert:

1. Wie verliefen Landnutzungsänderung und Siedlungsentwicklung in den St. Pöltener Traisenauen im Zeitraum 1870 bis 2000 ?

2. Was trieb die Siedlungsentwicklung in den Flussauen an? Welche Rolle spielten gewässerrelevante Faktoren wie Flussdynamik, Hochwasserrisiko und Hochwasserschutz? Welche Bedeutung hatten Bevölkerungswachstum, Stadtentwicklung und städtische Planungsziele?

Ausgehend von diesen Faktoren wird deutlich, wie die Siedlungsausdehnung in den Auen mit der Planung und dem tatsächlichen Ausbau des technischen Hochwasserschutzes sowie mit der Stadtentwicklung zusammenhing. In diesem Kontext ist auch die Frage nach zeitlichen Überlappungen oder deutlich erkennbaren asynchronen Mustern relevant, wie sie z.B. der Kosten-Nutzen-Rechnung im Hochwasserschutz entsprechen würden. In der abschließenden Diskussion wird schließlich das Beispiel St. Pölten mit anderen Gemeinden an der Traisen sowie mit der Stadtentwicklung Wiens in den Donauauen kurz verglichen.

Die historische Analyse der Siedlungsnutzung am Beispiel der St. Pöltener Traisenauen zeigt die enge und im 20. Jahrhundert kontinuierlich zunehmende Verflechtung zwischen Landnutzung und Siedlungsentwicklung in Flussauen und dem Ausbau des technischen Hochwasserschutzes und prinzi- 
pielle Muster des Hochwasserschutzes auf.

\section{Die Untere Traisen und St. Pölten als Untersuchungsregion}

Die Traisen ist einer der längsten $\mathrm{Zu}$ flüsse zur niederösterreichischen Donau. Von ihrem Ursprung in den niederösterreichischen Kalkalpen bis zur Mündung in die Donau entwässert sie ein Gebiet von $900 \mathrm{~km}^{2}$. Die Gesamtlänge des Flusses nach der Vereinigung der beiden Quellflüsse Unrechttraisen und Türnitzer Traisen beträgt $75 \mathrm{~km}$. Durch die Verlagerung des Hauptflusses in das Unterwasser des Donaukraftwerks Altenwörth in den 1970er-Jahren wurde der Flusslauf um sieben Kilometer länger. Mit der Renaturierung des Unterlaufs in den Jahren 2013 bis 2016 kam es zu einer neuerlichen Verlängerung um 4,5 km (s. zu diesem Projekt Eberstaller et al. (2018), in diesem Heft; Kaufmann et al. (2018), in diesem Heft).

Bei St. Pölten durchfließt die Traisen eine breite Talebene. Der mittlere Abfluss beträgt beim knapp $10 \mathrm{~km}$ flussauf von St. Pölten liegenden Pegel Windpassing $13,9 \mathrm{~m}^{3} / \mathrm{s}$. Bei einem 100jährlichen Hochwasserereignis fließen bei Windpassing $725 \mathrm{~m}^{3} / \mathrm{s}$ ab. Nicht nur dieses große Verhältnis zwischen Mittel- und Hochwasserabfluss, sondern auch das vergleichsweise hohe natürliche Gefälle von 3,5 bis 4,0\% im Mittelund Unterlauf bis ca. Traismauer sorgten für eine hohe flussmorphologische Dynamik. Durch die vielen Wildbäche im Einzugsgebiet ist zudem die Geschiebeführung über weite Strecken beträchtlich (Höbinger 1970).

Die Traisen war im Abschnitt von Wilhelmsburg bis Traismauer vor der Regulierung ein gewunden-verzweigter Fluss, der sich regelmäßig verlagerte und bis zu $600 \mathrm{~m}$ breit war. Bereits seit dem Mittelalter zweigte an der Unteren Traisen links- und rechtsufrig jeweils ein Mühlbach ab, wo die Wasserkraft weitgehend unabhängig von der Flussdynamik genutzt werden konnte.

St. Pölten liegt am Unterlauf der Traisen, unmittelbar nachdem der Fluss in die breite Talebene tritt. Im Jahr 2017 lebten hier mehr als 54.000 EinwohnerInnen. Die Stadt unterstand seit dem Ende des 15. Jahrhundert dem Landesfürsten und war bereits im Spätmittelalter ein wichtiger Wirtschaftsstandort im damaligen Erzherzogtum Österreich unter der Enns. Die Lage an der Traisen bot eine günstige Situation für Gewerbeansiedlungen und die Wasserkraftnutzung an den beiden linksund rechtsufrig verlaufenden Mühlbächen war gut ausgebaut. Spätestens seit dem 17. Jahrhundert organisierten sich die Mühlenbetreiber in genossenschaftlichen Verbänden, die gemeinsam die Wehre zur Wasserausleitung in die Mühlbäche unterhielten (Werneck 1965). Seit der Ernennung zum Bischofssitz im Jahr 1785 war die Stadt auch ein wichtiges kulturelles Zentrum Niederösterreichs (Gutkas 1982). Ab den 1860er-Jahren entwickelte sich St. Pölten zu einem Industriezentrum. Eine große Rolle spielte dabei die Anbindung an überregionale Bahnverbindungen. Seit 1986 ist St. Pölten die Landeshauptstadt von Niederösterreich.

Um die Siedlungsentwicklung St. Pöltens in den Traisenauen zu untersuchen, wurde der historische $\mathrm{HQ}_{100}-\mathrm{Ab}$ flussraum herangezogen. Dieser wurde durch eine hydraulische Modellierung ermittelt (Modellierung durch Ziviltechnikerbüro DonauConsult; HQ 100 -Wert für St. Pölten $740 \mathrm{~m}^{3} / \mathrm{s}$; s. auch Eberstaller et al. 2004; Fraiß 2004). Als Basis für die Hydraulik wurde anhand eines Laserscans mit einer Dichte von einem Punkt pro $\mathrm{m}^{2}$ ein Geländemodell erstellt. Um die Überflutungsflächen des 19. Jahrhunderts zu berechnen, wurden die heute bestehenden Uferbegleitdämme entfernt, sodass die Traisen bei einem modellierten $\mathrm{HQ}_{100}$-Abfluss ungehindert über die Ufer treten kann. Es wurde davon ausgegangen, dass das Längsgefälle der Traisen sowohl für die historischen als auch die aktuellen Berechnungen nahezu gleich war. Diese Annahme wird durch Gefälledaten eines technischen Berichts zur Traisenregulierung aus dem Jahr 1886 gestützt (Niederösterreichisches Landesbauamt 1886, Technischer Bericht). Die ermittelte $\mathrm{HQ}_{100}$-Fläche betrug für St. Pölten 313 ha.

\section{Die Traisenflusslandschaft im Bereich St. Pöltens vor der systematischen Regulierung}

Im 19. Jahrhundert war die Untere Traisen nach wie vor ein dynamischer Fluss und in den Auen wechselten Land- und Wasserzonen nach jedem größeren Hochwasser. Während das Gewässerbett im Ober- und Mittellauf weitgehend stabil war und die Ufer hoch genug, um ein zu häufiges Überfluten $\mathrm{zu}$ verhindern, wurden flussab der Gemeinde Traisen die Ufer suk- zessive niedriger. Uferanbrüche und Schotterablagerungen waren typisch. Ab Scheibmühl kam es häufig auch zu Verlagerungen des Gewässerbettes. Die höchste Dynamik zeigte der Flussabschnitt ab St. Pölten. Hier waren die Ufer sehr niedrig und im Gewässerbett gab es großflächige Schotterbänke und -inseln. In den 1880er-Jahren erreichte das eigentliche Flussbett bereichsweise über $200 \mathrm{~m}$ Breite. Das auf den Inseln und Ufern abgelagerte Material stammte vorwiegend von Seitenerosion (Niederösterreichisches Landesbauamt 1886). An engeren Stellen, wo der Abfluss weitgehend auf einen Hauptarm konzentriert war, gab es aber auch Tiefenerosion. An breiteren Stellen mit flachen Ufern wurde dieses Geschiebe wieder abgelagert. Flussauf von solchen Abschnitten kam es zu einer Stauwirkung, während flussab das $\mathrm{Ge}$ fälle steiler war. In Ablagerungsstrecken tendierte der Fluss dazu, seitlich auszuweichen oder er stürzte mit starkem Gefälle gegen ungeschützte Ufer, wie z. B. in der Strecke zwischen Altmannsdorfer und Spratzerner Wehr.

Von früheren lateralen Laufverlagerungen zeugten Reste alter Flussarme. Das Ausmaß von solchen Ereignissen wurde z.B. durch zwei Vermessungen der Jahre 1881 und 1886 dokumentiert. In dieser Zeit war der Lauf der Unteren Traisen an mehreren Stellen um 100 bis $200 \mathrm{~m}$ gewandert. Einen wesentlichen Anteil daran hatte wahrscheinlich das Hochwasser von 1882, für das kurz danach Abflussschätzungen gemacht wurden. Die Basis dafür waren Fließgeschwindigkeitsmessungen an der Eisenbahnbrücke in St. Pölten und Aufnahmen der Sohlrauigkeiten. Die damit berechneten Abflusswerte ergaben für das Ereignis 1882 einen Abfluss von $827 \mathrm{~m}^{3} / \mathrm{s}$ (Niederösterreichisches Landesbauamt 1886). Das würde einem Hochwasser über $\mathrm{HQ}_{100}$ entsprechen.

Erste größere Regulierungsmaßnahmen wurden an der St. Pöltener Traisen auf Kosten der Niederösterreichischen Stände zwischen 1817 und 1820 ausgeführt (Landesausschuss 1914). Diese Arbeiten konzentrierten sich auf die lokale Sicherung der Traisenufer bei der Brücke in St. Pölten. Bei Laufverlegungen war diese besonders gefährdet und eine Unterbrechung des Verkehrs und Handels war für die Stadt problematisch. Die Bauten wurden bei den Hochwässern 1821 und 1829 beschädigt und teils vernichtet. $\mathrm{Zu}$ dieser Zeit war ein erstes systematisches Regulierungs- 


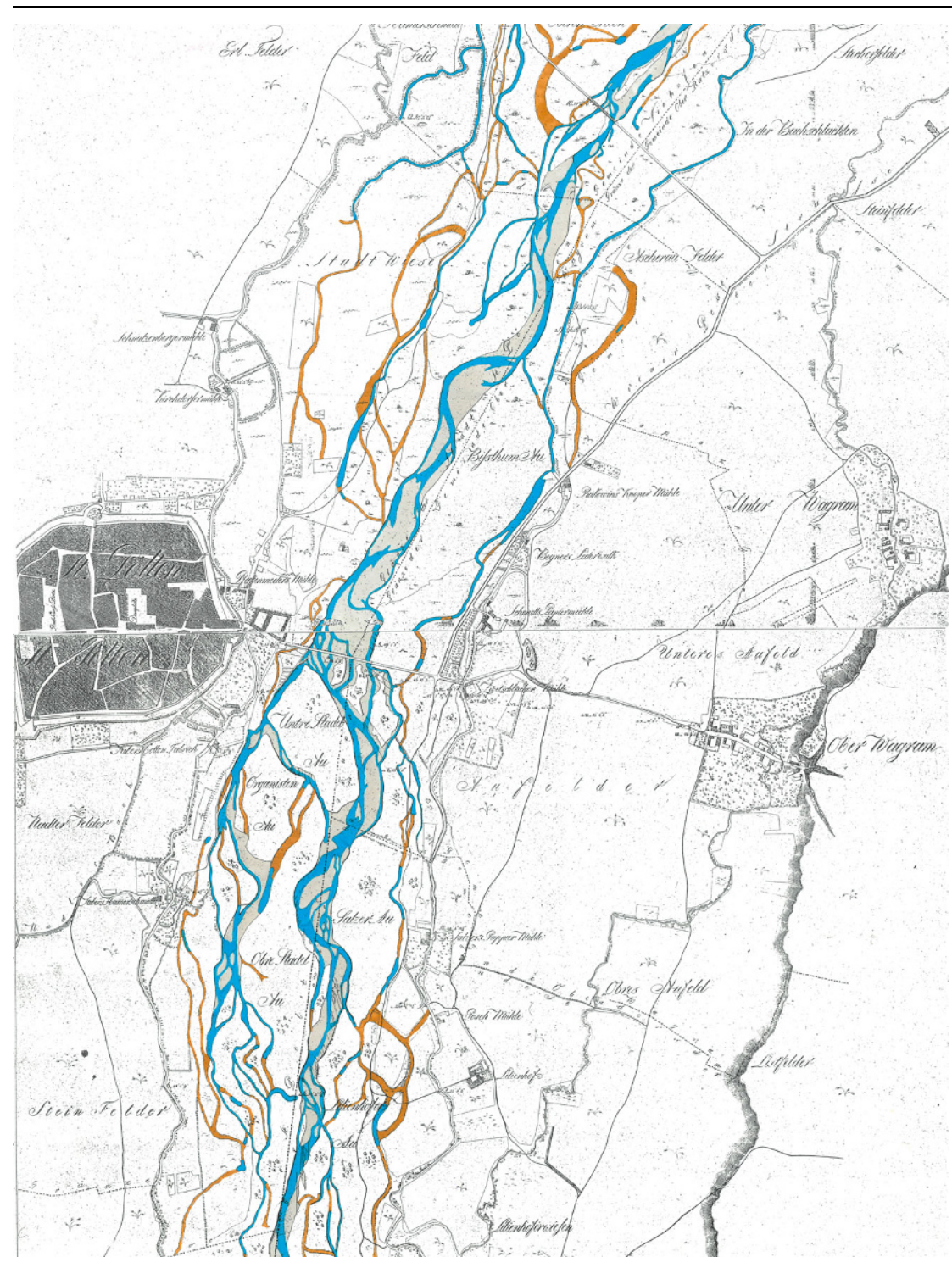

Abb. 1 DerunregulierteTraisenlaufimBereichvonSt.Pölten1825. Ausschnittaus dem Plan von Ignaz Dipolt „Der Traisenfluss mit seiner nächsten Umgebung von der Ochsenburger Brücke bis zur Donau zur Uibersicht reducirt aus der Aufnahme von den Jahren 1819 u. 1820 mit ausgemachter Trace der einzuleitenden Regulirung und der dieselbe bezwecken sollenden Baulichkeiten“ (Kopie des Instituts für Hydrobiologie, BOKU; Original in der NÖLB, B III/126. Kolorierung der Gewässerläufe durch die Autoren, blau: wasserführend bei $\mathrm{MQ}$, braun: bei $\mathrm{MQ}$ trockene Flussarme)

projekt an der Traisen bereits geplant (s. Abb. 1). Auch das Hochwasser 1839 erforderte im darauffolgenden Jahr die Erneuerung von Ufersicherungen, die bis in die 1860er-Jahre aus Faschinen bestanden. Für die Ausführung der Schutzbauten waren die jeweils anrainenden Gemeinden zuständig. triestandorte sowie Erholungsflächen und Kleingartensiedlungen.

Die aus den Jahren um 1870 stammende Wiederaufnahme („Reambulierung“) des Franziszeischen Katasters zeigt, dass in den St. Pöltener Traisenauen Auwald und Grünland dominierten. Ackerflächen gab es nur wenige in größerer Distanz zum Fluss. Im $\mathrm{HQ}_{100^{-}}$ Abflussraum standen lediglich acht Gebäude, die meisten davon waren Mühlen oder wassergebundene Gewerbebetriebe. Von insgesamt 14,1 ha bebauter Fläche entfielen 18707 ha auf die Trasse der Westbahn bzw. den St. Pöltener Bahnhof, etwas mehr als 1 ha auf Industriebetriebe und knapp 6 ha auf Wohnflächen. Das Siedlungsareal St. Pöltens beschränkte sich im Wesentlichen noch auf den historischen Stadtkern (s. Tab. 1, Abb. 2). Die Analyse der Katasterpläne aus den Jahren 1930 bis 1935 zeigte, dass zwischen 1870 und 1930 eine erste Ausdehnung St. Pöltens in den HQ100-Abflussraum stattfand. Dieser beschränkte sich allerdings auf den äußeren Rand der überflutungsgefährdeten Zone. Unmittelbar entlang des Flusses verblieb an beiden Ufern Auwald. Anhand einer topografischen Karte von 1907 lässt sich abschätzen, dass die meisten Gebäude erst danach errichtet wurden. Die Stadt St. Pölten war in den 60 Jahren zwischen 1870 und 1930 beträchtlich gewachsen. Die Siedlungsausdehnung erfolgte jedoch vor allem südlich und nördlich, teils auch westlich des historischen Stadtkerns am linken Traisenufer (s. Abb. 2).

Zwischen 1930 und 1960 dehnten sich Gebäude zunehmend zum traisennahen Überflutungsraum hin aus. Es handelte sich vor allem um Kleingartensiedlungen. Nach wie vor wuchs die Stadt vor allem außerhalb der hochwasserexponierten Zonen, in den drei Jahrzehnten zwischen 1930 und 1960 $\mathrm{zu}$ einem erheblichen Teil auch am rechten Flussufer. Die Errichtung dauerhafter Wohnhäuser unmittelbar an der Traisen setzte vor allem im Zeitraum 1960 bis 1980 ein und erstreckte sich zunächst wiederum vor allem auf das linke, zentrumsnähere Ufer. In dieser Phase war das Wachstum der Stadt gemessen an der Zahl der jährlich neu errichteten Gebäude im Überflutungsraum erstmals gleich hoch wie außerhalb. Von 1980 bis 2000, nach der Erhebung St. Pöltens zur Landeshauptstadt und der Fertigstellung des Regierungsviertels, nahm die Bebauung innerhalb des Überflutungsraumes 
Tab. 1 Entwicklung der Siedlungsflächen (Gebäude, Infrastruktur, Industriestandorte) in den St. Pöltener Traisenauen ( $\mathrm{HQ}_{100^{-}}$ Überflutungsfläche) im Zeitraum 1870 bis 2000

\begin{tabular}{|c|c|c|c|c|c|}
\hline & 1870 & 1930 & 1960 & 1980 & 2000 \\
\hline Gebäude, Infrastruktur, Industriestandorte & 14,1 & 96,9 & 183,7 & 192,6 & 206,1 \\
\hline
\end{tabular}
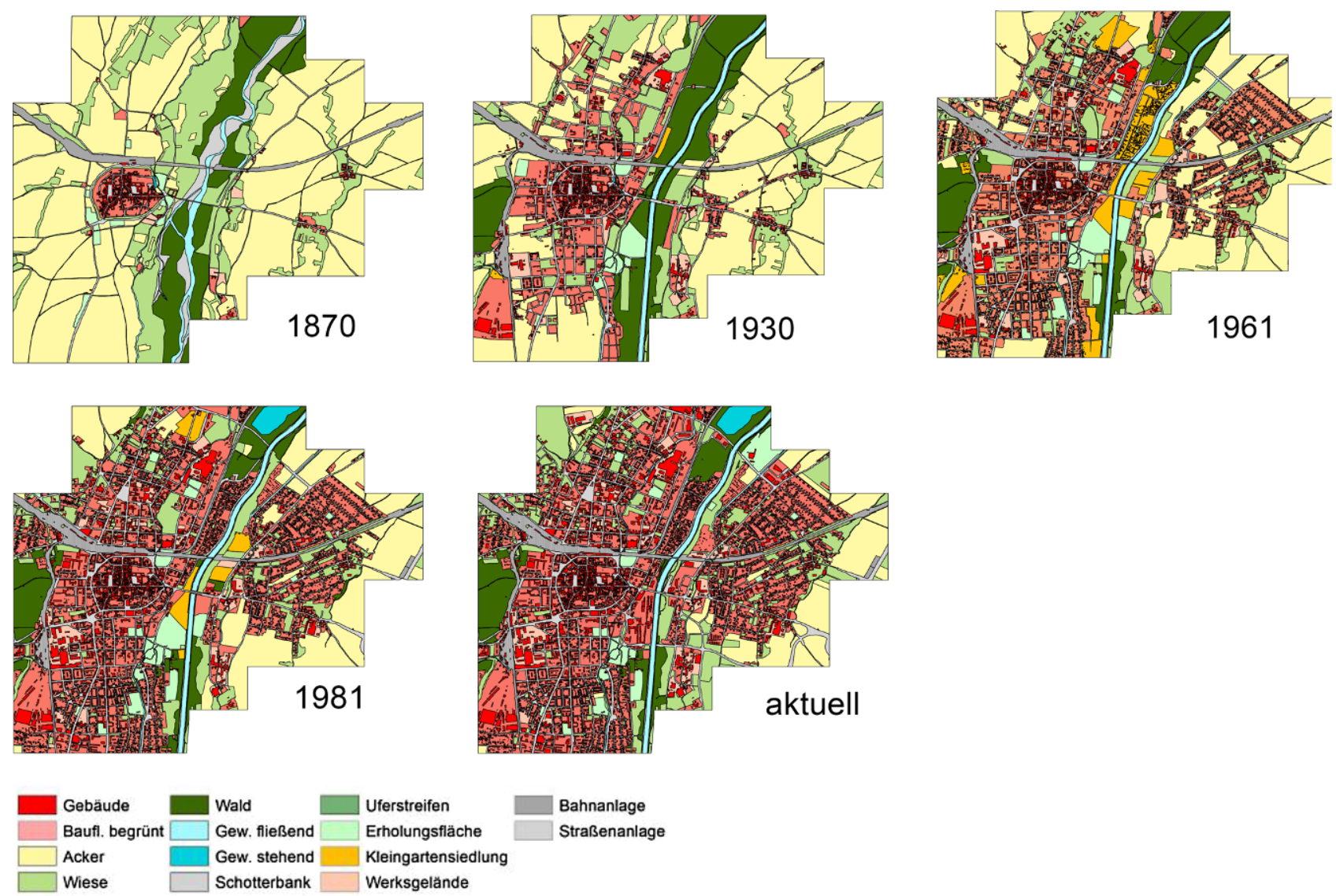

Abb. 2 Entwicklung derLandnutzungund Siedlungszonen in denSt. PöltenerTraisenauen(Digitalisierung der Landnutzung B. Fraiß, s. Eberstaller et al. (2004) sowie Fraiß (2004))

sowohl am linken als auch am rechten Traisenufer weiter zu. Im Jahr 2000 erstreckte sich das Siedlungsgebiet auf ca. 206 der insgesamt 313 ha (s. Tab. 1 sowie Abb. 2 und 3).

Insgesamt stieg der Anteil höherwertig genutzter Flächen in der $\mathrm{HQ}_{100^{-}}$ Zone von lediglich 4,3\% im Jahr 1870 auf $62,8 \%$ im Jahr 2000. Besonders hoch war die Siedlungsausdehnung zwischen ca. 1910 und 1930 auf knapp 30\% der gesamten Fläche sowie zwischen 1930 und 1960 auf ca. $56 \%$. Bis 1980 erhöhte sich das Ausmaß nur mehr gering auf 58,5\%, zwischen 1980 und 2000 nochmals um wenige Prozent. Der Anteil an Äckern, Grünflächen und Auwald nahm parallel zur Ausdehnung der Siedlungen ab. Im Jahr 2000 war der Überflutungsraum in Zentrumsnähe bzw. um das Regierungsviertel vollstän- dig verbaut. Es waren nur mehr wenige Grünland- und Waldflächen verfügbar, deren Erhaltung teils durch Flächenwidmungspläne gesichert war. Im Norden gab es jedoch auch potenzielle Betriebszonen. Am rechten Traisenufer existierten sowohl zwischen dem Primär- und Sekundärdamm der Traisen als auch außerhalb der Sekundärdämme noch größere Waldstandorte und Grünflächen. Letztere waren teils als Grünland gewidmet, teils auch als potenzielle Wohn- und Industriegebiete. Insgesamt waren im Jahr 2000 im historischen $\mathrm{HQ}_{100}$-Abflussraum noch $25 \%$ an nicht bebauten Flächen vorhanden (s. Abb. 3).

Der Landnutzungswandel von einem analysierten Zeitschnitt zum nächstfolgenden zeigt ebenfalls, dass die größten Änderungen zwischen 1870 und 1930 bzw. zwischen 1930 und 1960 stattfanden (s. Abb. 4). Zwischen 1870 und 1930 entstanden auf früheren Gewässerflächen Auwälder (s. Abb. 4a), wohl als unmittelbare Folge der Regulierung und Verlandung bzw. aktiven Verfüllung von Altwässern (s. unten Kapitel zur Traisenregulierung). Neue Siedlungsflächen und Infrastrukturanlagen wurden überwiegend auf ehemaligen Grünflächen errichtet. Zwischen 1930 und 1960 wurden für neu bebautes Gebiet auch traisennah liegende Auwälder gerodet, vorwiegend für die neuen Kleingartenanlagen (Abb. 4b). 


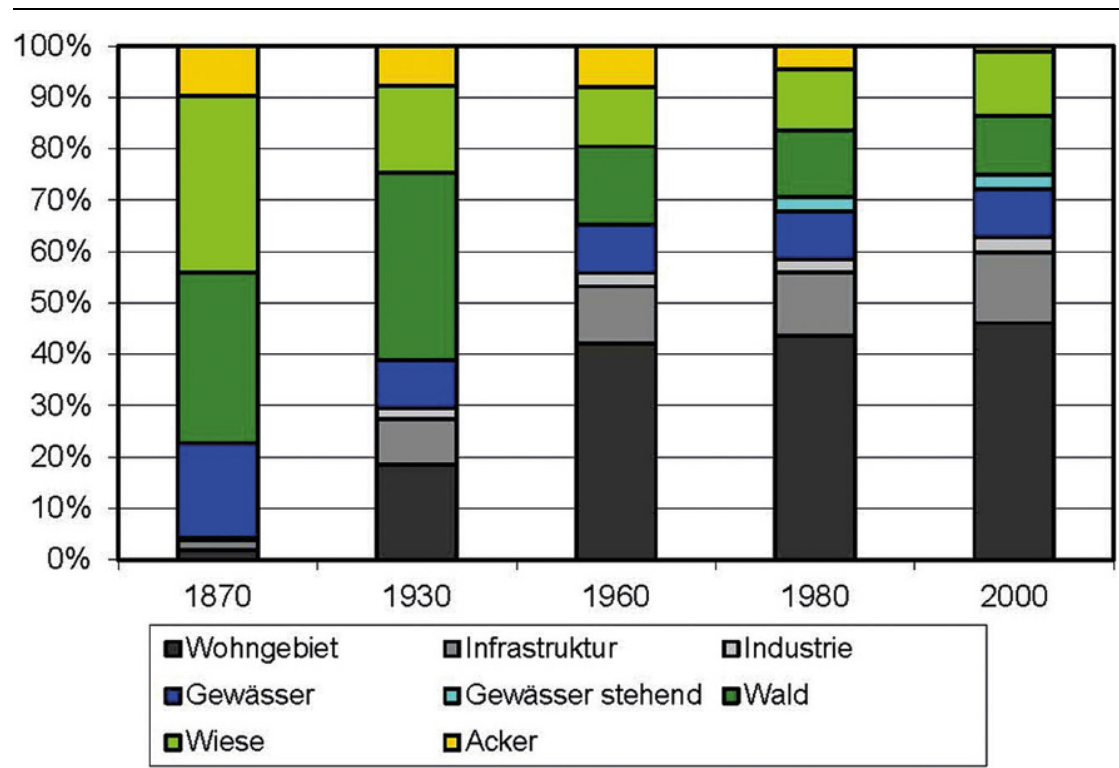

Abb. 3 ProzentuelleVeränderung der Flächennutzung in den St. Pöltener Traisenauen (historischer $\mathrm{HQ}_{100}$-Abflussraum; Fläche ca. 313ha)

5 Demografie als Treiber der Siedlungsentwicklung und Stadtplanung als Steuerungsinstrument

Mit der Integration St. Pöltens in das überregionale Bahnnetz wurde eine wichtige Voraussetzung für die Entwicklung der Stadt zu einem niederösterreichischen Industriezentrum geschaffen. Die größte Rolle spielte die Anbindung an die 1858 zwischen Wien und Linz und 1860 zwischen Linz und Salzburg eröffnete Westbahn. In den folgenden Jahrzehnten wurde St. Pölten mit anderen Hauptbahnlinien vernetzt und es wurden neue Nebenlinien geschaffen. Zur Südbahn existierte ab 1877 eine Verbindung über Leobersdorf. Die Anbindung zur Franz-Josefs-Bahn über Herzogenburg und Tulln wurde 1885 eröffnet. Nebenlinien bestanden ab 1878 nach Lilienfeld bzw. Schrambach. Der Ausbau dieser Linie nach Kernhof wurde 1893 abgeschlossen, eine Abzweigung von Freiland nach Türnitz bis 1908 realisiert (Stein 1928). Die Strecke von St. Pölten nach Kirchberg an der Pielach wurde 1898 eröffnet; eine Netzerweiterung nach Mariazell bzw. Gußwerk bis 1907 fertiggestellt (Stokreiter 1988).
Die ausgezeichneten Verkehrsverbindungen sowie vor allem seit dem Beginn des 20. Jahrhunderts die Errichtung von Wasser- und Dampfkraftwerken zur Stromerzeugung lösten die traditionellen Protoindustriegründungsmuster des Agrarzeitalters auf. Die lokale Verfügbarkeit von Wasserkraft war nicht länger ein Standortvorteil, sondern vielmehr die Nähe zu Verbrauchs- und Handelszentren oder eine gute Verkehrsvernetzung mit denselben. Durch die Errichtung von Elektrizitätskraftwerken in Verbindung mit dem gut ausgebauten Bahnnetz konnte sich St. Pölten hier im Vergleich mit anderen niederösterreichischen Städten entscheidende Wettbewerbsvorteile sichern.

Bis 1860 waren in St. Pölten mit Ausnahme der Salzerschen Papierfabrik alle frühindustriellen Betriebe und Gewerbe aufgelöst. Gleichzeitig entstanden zahlreiche neue Unternehmen, so z.B. 1859 die Harlander Zwirnfabrik, 1860 die Börtelfabrik Leidenfrost, 1870 die Revolverfabrik Gasser (später Weicheisen- und Stahlguss AG; 1930 aufgelassen) oder 1882 die Seifenfabrik Benker (Gutkas 1982). Im Jahr 1903 erfolgte die erste große, moderne Industriegründung durch die Firma Voith, die ihre Produktion 1904 mit einem Stand von 200 Mitarbeitern aufnahm (Zand 1997). Zur gleichen Zeit wurde die Firma Glanzstoff gegründet, die 1906 mit insgesamt 306 Mitarbeitern in Betrieb ging, bereits zwei Jahre später ihre Werksanlagen vergrößerte und 192870 Angestellte und 2950 Arbeiter hatte. Schließlich wurde 1907
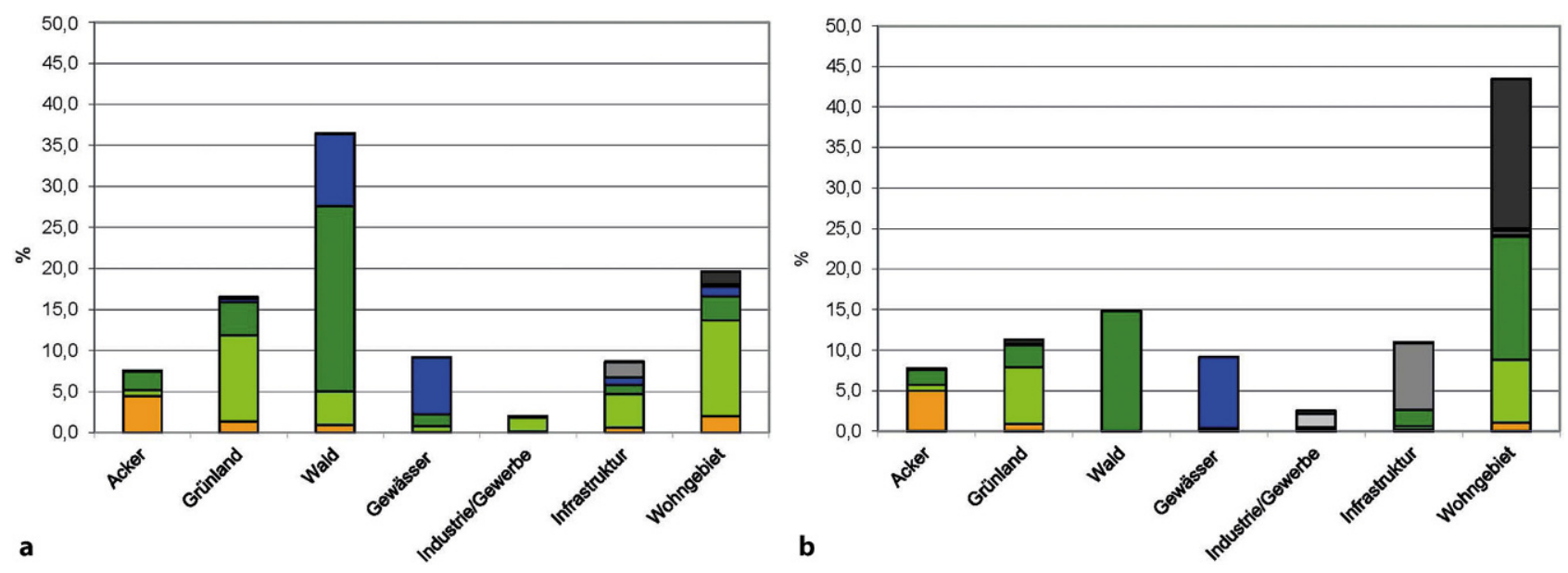

Abb. 4 Landnutzungswandel(Turn Over derFlächen) in den St. Pöltener Traisenauen(HQ100-Abflussraum); a Veränderung von 1870 bis 1930; b von 1930 bis 1960. Die Balken zeigen, aus welchen Landnutzungskategorien die Nutzungstypen 1930 bzw. 1960 hervorgegangen sind. Farben: orange Äcker, hellgrün Grünland, dunkelgrün Wälder, blau Gewässer, hellgrau Industrie/Gewerbe, mittelgrau Infrastruktur, dunkelgrau Siedlungsgebiet 
die Hauptwerkstätte der Staatsbahnen eröffnet.

Die Industrialisierung initiierte eine starke Nachfrage nach Arbeitskräften, die auch große Abwanderungsbewegungen von den ländlichen Gebieten in die Stadt St. Pölten auslösten (Gutkas 1982). Die demografische Entwicklung war dementsprechend dynamisch. Im letzten Drittel des 19. Jahrhunderts, zwischen 1869 und 1900, stieg die Bevölkerung innerhalb der aktuellen Stadtgrenzen St. Pöltens um $70 \%$ von 14.470 auf 24.507. Zwischen 1869 und 1910 verzeichnete die Stadt den größten Bevölkerungszuwachs aller niederösterreichischen Gemeinden (Stokreiter 1988). Vor dem Ersten Weltkrieg erreichte die EinwohnerInnenzahl 35.648, bis zum Zweiten Weltkrieg erfolgte im Vergleich zu 1900 annähernd eine Verdoppelung auf knapp 49.000 Personen. Mit Ausnahme eines Einbruchs nach dem Zweiten Weltkrieg schwankte die Bevölkerungszahl nach 1950 nur mehr in geringem Ausmaß.

Die Demografie in Verbindung mit der guten Konjunkturentwicklung der Industriebetriebe erzeugte einen enormen Wohnungsbedarf, der von Beginn an durch kostengünstige Wohnbauten abgedeckt und gesteuert werden sollte. Der 1903 gegründete „Verein zur Erbauung billiger Wohnungen“ schuf eine erste „Arbeiterwohnkolonie“. Das rasche Wachstum der Firma Glanzstoff förderte die Nachfrage nach zusätzlichem Wohnraum. Auf insgesamt $60.000 \mathrm{~m}^{2}$ Fläche wurden zunächst 16 Wohnbauten errichtet. Bis 1928 stieg die Zahl auf 77 Gebäude mit 89 Wohnungen (Stein 1928; Zand 1997). Industriebetriebe beteiligten sich aktiv an den Wohnbauprogrammen und gehörten teils auch dem „Verein zur Erbauung billiger Wohnungen“ an. Neben der Firma Glanzstoff errichteten auch die Voithwerke auf ihrem Gelände auf ca. $30.000 \mathrm{~m}^{2}$ eine Siedlung (Voithsiedlung, Stein 1928). Im Jahr 1914 besaß die Firma 28 Gebäude mit 234 Wohnungen, 1928 bereits 38 Häuser mit 381 Wohnungen und 40 Einfamilienhäuser. Eine 1921 neu gegründete Wohnungsgenossenschaft schuf zwischen 1921 und 1931 zahlreiche weitere Wohnhäuser (Stein 1928; Zand 1997).

St. Pölten setzte bereits im 19. Jahrhundert auf eine gezielte Stadtplanung zur Steuerung der Siedlungs- und Bautätigkeit und initiierte die Ausarbeitung von Stadterweiterungsplänen. Die Siedlungsausdehnung konzentrier- te sich nach der Schleifung der Stadtmauer 1854 zunächst um den historischen Stadtkern. Um sicherzustellen, dass die absehbar weiter starke Bautätigkeit geregelt verläuft, stellte der Gemeinderat bereits 1872 einen „Regulierungs- und Stadterweiterungsplan" in Aussicht. Die Ausschreibung verzögerte sich allerdings bis 1883. Der schließlich 1886 fertig gestellte „Regulierungsplan" sah die Siedlungserweiterung vor allem südwestlich und nördlich des Stadtkerns vor. Eine Abgrenzung oder spezielle Ausweisung von Grünflächen zur Erholung war darin noch nicht enthalten. Erst 1929 wurde ein eigener Grünzonenplan für St. Pölten erlassen. $\mathrm{Zu}$ diesem Zeitpunkt war die erste systematische Regulierung der Traisen abgeschlossen (s. unten Kapitel zur Traisenregulierung). Die nach wie vor bestehenden Überflutungszonen entlang der Traisen wurden darin als bevorzugte Flächen für Erholungsnutzung ausgewiesen und durften nicht bebaut werden. Auch im Flächenwidmungs- und Bebauungsplan von 1936 befanden sich die künftigen Bauzonen vorwiegend nördlich, westlich und südlich des existierenden Siedlungsgebiets sowie in den nunmehr eingegliederten Stadtteilen Ober- und Unterwagram am rechten Traisenufer. Die entlang der Traisen bestehenden und im Grünzonenplan für die Erholung gewidmeten Flächen wurden 1936 übernommen und als solche nicht zuletzt aufgrund der regelmäßigen Überflutung durch die Traisen beibehalten.

Obwohl die Bebauungspläne nicht in allen Punkten umgesetzt wurden, zeigen sie nicht nur die Motive der gesteuerten Entwicklung der Besiedlung, sondern vor allem auch die bewusste Freihaltung einer Überschwemmungszone entlang der Traisen. Erst um 1960 wurden diese Grundsätze aufgegeben. Die Traisenauen wurden nun aktiv in die städtischen Siedlungsentwicklungszonen aufgenommen. Diese Entscheidung stand in Zusammenhang mit neuen Hochwasserschutzprinzipien an der Traisen.

\section{Hochwasserschutz als Treiber der Siedlungsentwicklung in den Traisenauen}

Nachdem bis in die 1860er-Jahre Regulierungen an der Traisen lediglich lokal existierten und sich meist auf den Uferschutz von wichtigen Abschnitten beschränkten, ordnete die Bezirkshaupt- mannschaft St. Pölten im Jahr 1872 die Ausarbeitung eines neuen Traisenregulierungsprojekts von Wilhelmsburg bis zur Donaumündung an. Die im folgenden Jahr für die Ausführung und Erhaltung von $\mathrm{zu}$ bauenden Schutzbauten gegründeten Wassergenossenschaften forderten eine Abdeckung der späteren Instandhaltungskosten aus Landesmitteln. Die Ablehnung dieser Forderung brachte das Vorhaben ins Stocken (Herrmann 1930). Es blieb weiterhin bei lokalen und regionalen Arbeiten an bestehenden Sicherungsanlagen, obwohl in den Jahren 1879, 1882 und 1886 weitere systematische Regulierungsprojekte gemacht wurden (Landesausschuss 1914).

Keines der im 19. Jahrhundert geplanten Hochwasserschutzprojekte wurde verwirklicht. Erst als durch das Hochwasser 1903 das Stadtzentrum von St. Pölten bedroht war, wurde die systematische Regulierung der Unteren Traisen bis Traismauer in Angriff genommen und von 1904 bis 1913 ausgeführt. Der Abschnitt entlang der Stadt St. Pölten wurde in den Jahren 1905 bis 1909 realisiert (Herrmann 1930). Im Zuge der Regulierung wurde zwischen Wilhelmsburg und Traismauer ein Trapezprofil für einen Abfluss von $400 \mathrm{~m}^{3} / \mathrm{s}$ gebaut (ca. $\mathrm{HQ}_{10}$ ). Die Profilbreite betrug zwischen dem Spratzerner Wehr und Traismauer $50 \mathrm{~m}$. Über weite Strecken wurde ein neues Gewässerbett geschaffen und mit dem Aushub wurden die Nebengewässer verfüllt. Da Untersuchungen zeigten, dass Strecken mit einem Gefälle von $2 \%$ eine stabile Sohlenlage hatten, reduzierte man mithilfe von zahlreichen 40 bis $100 \mathrm{~cm}$ hohen Sohlstufen das Gefälle auf diesen Wert. Die Böschungsneigung sollte einheitlich 1:5 betragen, die Sicherung des Böschungsfußes erfolgte entweder mit Betonwänden oder Weidenflechtzäunen. Die Böschungen selbst wurden mit einer $20 \mathrm{~cm}$ starken Humusdecke und Rasenbelag gesichert. Entlang der Traisen befand sich ein $4 \mathrm{~m}$ breiter "Wirtschaftsstreifen" (= Treppelweg), landeinwärts davon wurden auf einem $14 \mathrm{~m}$ breiten Streifen Weidenstecklinge angepflanzt, die Hochwasserschäden verringern sollten (Landesausschuss 1914).

Ziel dieser Regulierung war es nicht, die Auen für eine zu erwartende Siedlungsausdehnung vor regelmäßigen Überschwemmungen zu schützen. Vielmehr sollten die an den Mühlbächen befindlichen Industriebetriebe oder 

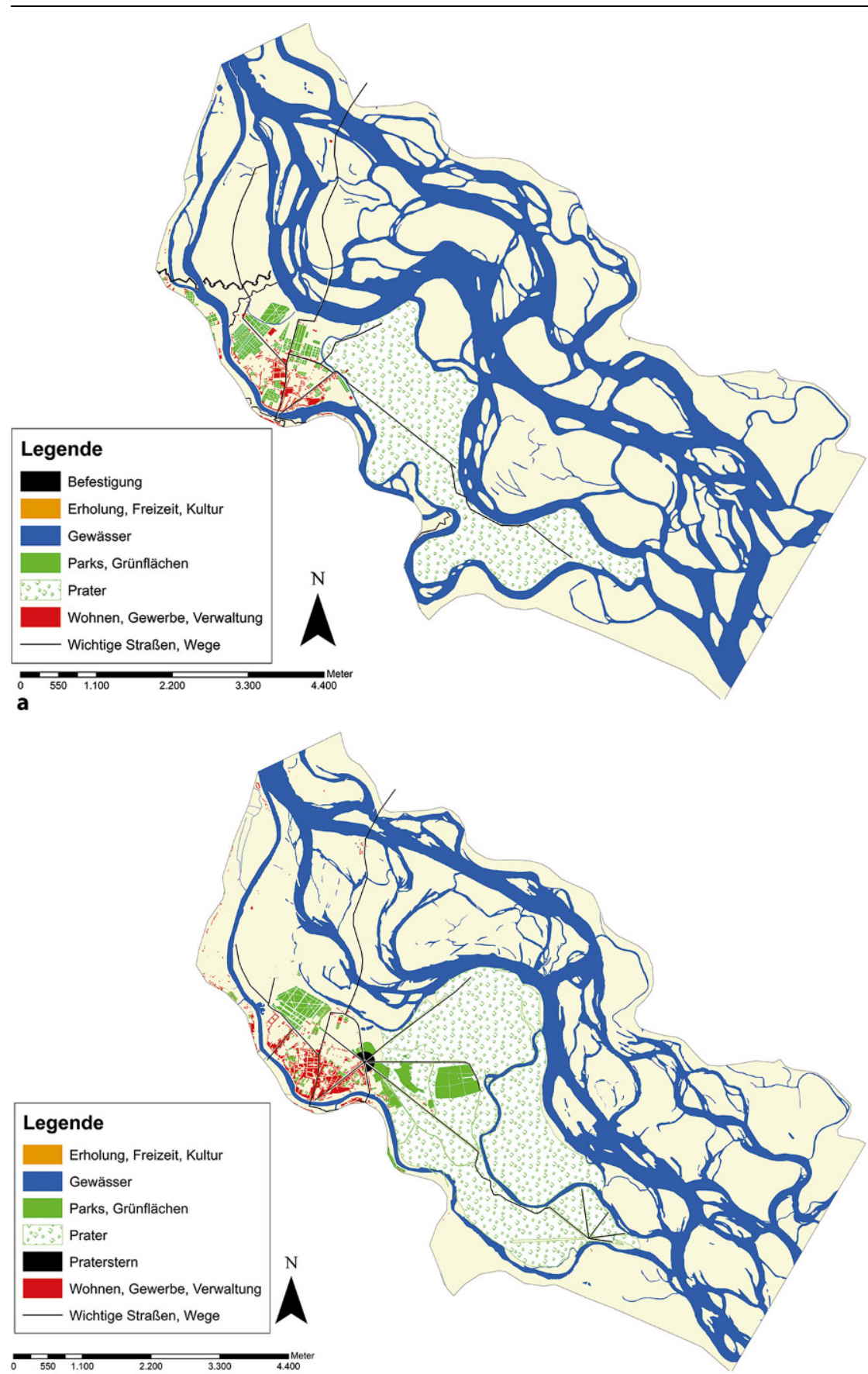

b

Abb. 5 Siedlungsentwicklung in den Wiener Donauauen in den Jahren 1704, 1875, 1912 und 2007. Die „Aktive Zone“ ist die seit ca. 1500 flussmorphologisch dynamische Zone (s. Hohensinner et al. 2013)

flussnahe gelegene Orte (z.B. Einöd) vor Hochwasserschäden bewahrt werden. Der Hochwasserschutz wurde bewusst nur auf häufig wiederkehrende Ereignisse ausgelegt (Landesausschuss 1914). Für größere und damit seltener auftretende Ereignisse erachteten die Planer die bestehenden freien Flächen entlang der Traisen als ausreichend.
$21 \mathrm{~km}$ wieder instandgesetzt, das Gefälle durch weitere Sohlstufen auf 1,5\%o reduziert und die Hochwasserschutzdämme für einen Abfluss von $520 \mathrm{~m}^{3} / \mathrm{s}$ ausgebaut (Zehndorfer 1970). Das entspricht nahezu einem $\mathrm{HQ}_{30}$, das aktuell für den Pegel Windpassing mit $540 \mathrm{~m}^{3} / \mathrm{s}$ angegeben wird. Flussab von St. Pölten liegende Teilstrecken wie Ratzersdorf, Herzogenburg oder St. Andrä wurden zum Teil erst nach dem Zweiten Weltkrieg saniert.

Beim Hochwasser 1959 erreichte die Traisen bordvollen Abfluss, der somit auf knapp $520 \mathrm{~m}^{3} / \mathrm{s}$ geschätzt wurde. Als Folge wurde 1960 schließlich der Ausbau der Traisenregulierung auf ein $\mathrm{HQ}_{100}$ zur Maxime. Entsprechend den Vorgaben für das gleichzeitig beauftragte Regulierungsprojekt war erstmals explizit eine neue Widmung von Bauzonen direkt entlang der Traisen zu berücksichtigen. Die Planung wurde 1963 abgeschlossen (Deiss 1963). Als erstes umgesetzt wurde der Bauabschnitt südlich der A1, d.h. flussauf des hier analysierten Bereichs. Im unmittelbaren Untersuchungsabschnitt wurde zwischen 1982 und 1987 die Strecke zwischen Autobahn- und Bundesstraßenbrücke (B1) reguliert. Im nördlich gelegenen Teil erfolgte der Ausbau bis zur Adolf-Schärf-Brücke teilweise erst im Zuge der Errichtung des Regierungsviertels St. Pölten und wurde 1996 abgeschlossen. In Verbindung mit der Errichtung des Regierungsviertels wurden sie teilweise auf das rechnerische Höchsthochwasser von $1100 \mathrm{~m}^{3} / \mathrm{s}$ ausgelegt.

\section{Siedlungsentwicklung in den}

St. Pöltener Traisenauen als Interaktion zwischen Industrialisierung, Urbanisierung und Hochwasserschutzpraktiken des 20. Jahrhunderts

Die integrative Analyse des räumlichen und zeitlichen Verlaufs der Siedlungsentwicklung St. Pöltens in den Traisenauen zeigt, dass Industrialisierung, Urbanisierung und Hochwasserschutzpraktiken des 20. Jahrhunderts treibende Faktoren waren und Wechselwirkungen existierten. Die wirtschaftliche Entwicklung der Stadt seit den 1860erJahren ging mit steigender Bevölkerung und der Nachfrage nach neuen Wohnund Gewerbestandorten einher. Diese Zonen entstanden vorwiegend im Süden, Westen und Norden des historischen Stadtzentrums. Trotzdem dehnte 


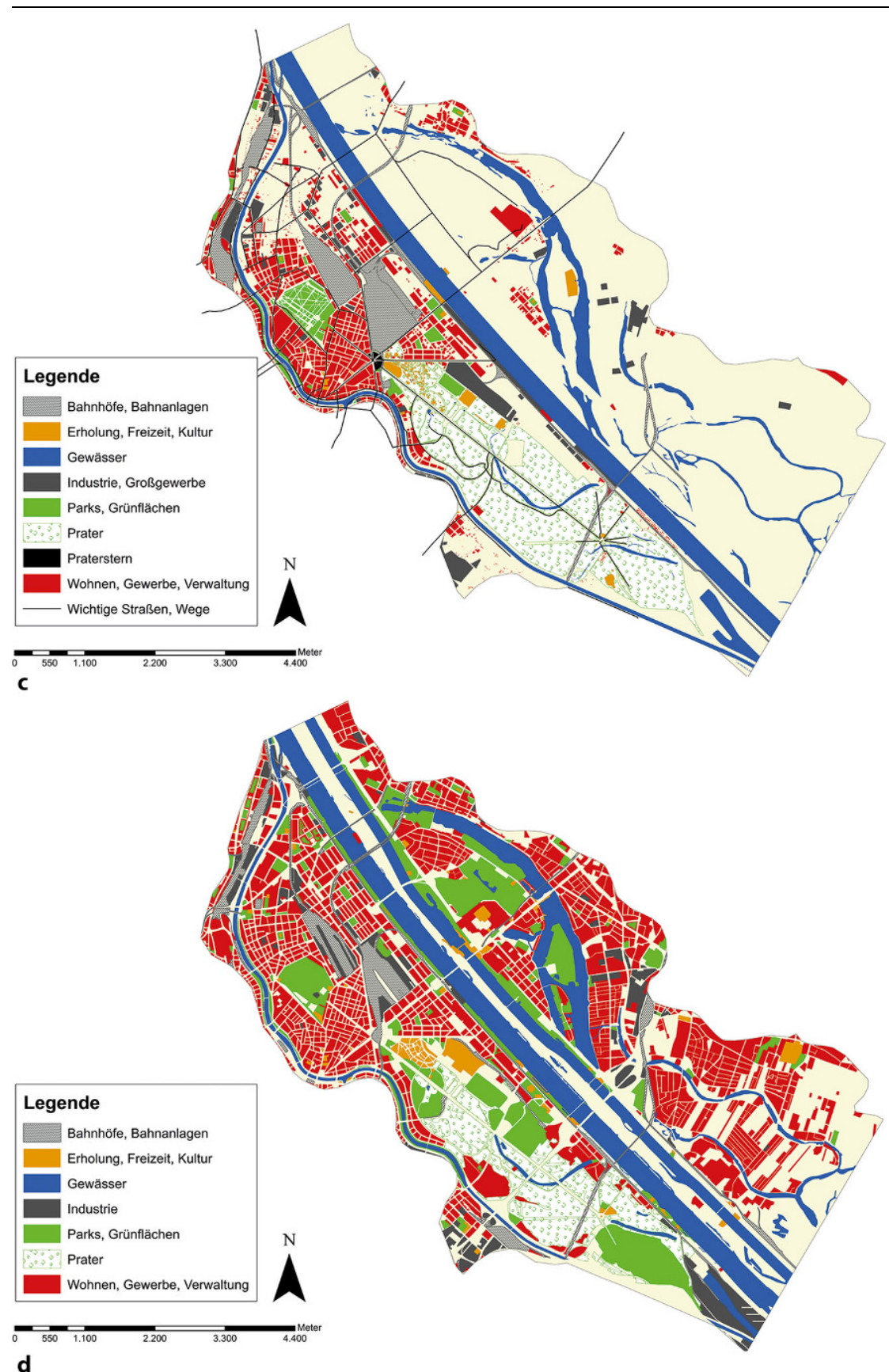

Abb. 5 (Fortsetzung)

sich die Stadt im 20. Jahrhundert kontinuierlich in die Traisenauen aus. Die Expansion begann zunächst linksufrig und beschränkte sich auf die Ränder des $\mathrm{HQ}_{100}$-Abflussraums. Entlang der seit 1909 existierenden Hochwasserschutzdämme verblieb bis 1960 ein Grünland- bzw. Auwaldstreifen, der durch städtische Planungsinstrumente von höherrangiger Bebauung freigehalten wurde. Bis 1960 entstanden hier allerdings nicht dauerhaft bewohnte Kleingartensiedlungen. Nach dem
Aussicht auf den künftigen Hochwasserschutz reichte für die Bauentscheidungen offensichtlich aus. Bis 1980 war im untersuchten $\mathrm{HQ}_{100}$-Abflussraum ein Schadenspotenzial von knapp 33Mio. $€$ entstanden (s. Eberstaller et al. 2004). Dass Überflutungsschäden ausblieben, war einer längeren Phase ohne Hochwässer zu verdanken und weniger einer entsprechenden Vorsorge. Beim Jahrhunderthochwasser vom Juli 1997 verhinderten die kurz davor fertiggestellten Dämme Schäden.

Das Beispiel der Ausdehnung der Stadt St. Pölten in die Traisenauen ermöglicht es, die Motive der Besiedlung von Flussauen anhand von Hochwasserschutz- und Stadtentwicklungsplanung und entsprechende Wechselwirkungen explizit nachzuvollziehen. In Lilienfeld und Pottenbrunn, zwei weiteren Kommunen entlang der Traisen, deren Siedlungsentwicklung im Rahmen des Forschungsprogramms „Flood Risk" untersucht wurde (s. Eberstaller et al. 2004), verlief die Kolonisierung der Auen ohne übergeordnete Steuerung und die Motive sind nicht so explizit dokumentiert. Trotzdem lassen sich anhand der tatsächlich erfolgten Bebauung des $\mathrm{HQ}_{100}$-Abflussraums einige weitere Schlussfolgerungen in Hinblick auf wichtige Treiber ziehen. Lilienfeld liegt im Mittellauf der Traisen in einem engen Sohlental. Im Jahr 2017 hatte die Stadtgemeinde knapp 2900 EinwohnerInnen. Das Stift Lilienfeld machte die Region früh zu einem kulturellen Zentrum. Wasserkraft und Waldreichtum begründeten die vor allem bis zum Ende des 19. Jahrhunderts vergleichsweise große wirtschaftliche Bedeutung. Heute gibt es mit wenigen Ausnahmen keine größeren Industriebetriebe. Lilienfeld erlebte am Ende des 19. Jahrhunderts keinen mit St. Pölten vergleichbaren industriellen Aufschwung und verzeichnete im 20. Jahrhundert keinen nennenswerten Anstieg der Bevölkerung. Um 1870 lag das bebaute Gebiet überwiegend außerhalb des Überflutungsraums und konzentrierte sich um die auf einem Plateau über dem Fluss liegenden Stiftsgebäude. Im 20. Jahrhundert war die Ausdehnung der Siedlungsflächen in den Traisenauen jedoch meist wesentlich größer als auf den Hanglagen, obwohl ein systematischer Hochwasserschutz erst zwischen 1931 und 1934 bzw. 1946 bis 1948 geschaffen wurde. Auch in Lilienfeld wuchsen die Wohn- und Gewerbezonen sowie Infrastrukturanlagen im Überflutungs- 
raum vor allem bis 1960 stark, danach verlangsamte sich die Entwicklung. Das Beispiel Lilienfelds zeigt, dass bei der Siedlungsentwicklung entlang von Flüssen neben dem Hochwasserrisiko auch Faktoren wie Geländetopografie und Erschließung über Flussachsen eine Rolle spielen. Man siedelte im Überflutungsraum, wenn leicht zugängliche Flächen außerhalb knapp waren. Diese Vermutung stärkt auch das Beispiel Pottenbrunn. Diese mittlerweile zu St. Pölten gehörige Katastralgemeinde mit aktuell knapp 2500 EinwohnerInnen liegt ebenfalls in einem breiten Talraum am Unterlauf der Traisen. Das Bevölkerungswachstum war im späten 19. und im 20. Jahrhundert gering. Bis 1970 gab es keine größeren Industriebetriebe. Seit der systematischen Traisenregulierung zwischen 1904 und 1913 bestand ein Hochwasserschutz, der im Verlauf weiterer Maßnahmen zwischen 1959 und 1973 von $\mathrm{HQ}_{10}$ auf ca. $\mathrm{HQ}_{25}$ verbessert wurde. Eine Siedlungsausdehnung in den Traisenauen erfolgte über den gesamten Zeitraum seit 1870, im Vergleich mit St. Pölten und Lilienfeld jedoch nur in einem geringen Ausmaß und an konzentrierten Flächen an den äußeren Rändern des $\mathrm{HQ}_{100}$-Abflussraums.

In anderen Flussauen entlang stark wachsender Siedlungszentren, für die in den letzten Jahren die Landnutzungsentwicklung im Detail untersucht wurde, verlief die Entwicklung im 20. Jahrhundert ähnlich wie an der Traisen in St. Pölten. Ein zwar um Dimensionen größeres aber in der Sache durchaus vergleichbares Beispiel stellt die Stadt Wien dar. Hier waren die Donauauen bis ins 19. Jahrhundert weitgehend unbesiedelt. Eine Ausnahme stellte lediglich die bereits seit dem 14. Jahrhundert bestehende Siedlung „Unterer Werd“ dar, die bis ins 19. Jahrhundert zwar kontinuierlich, aber insgesamt wenig bzw. räumlich konzentriert wuchs. Erst ab den 1830er-Jahren dehnte sich die mittlerweile Leopoldstadt genannte Gemeinde weiter in Richtung Norden und Nordosten aus (heutiger Wiener Bezirk
Brigittenau). Die zunächst nicht organisierte Bautätigkeit wurde ab 1861 durch einen von der Stadtverwaltung beauftragten Bebauungsplan geregelt. Zu dieser Zeit war die Bevölkerung Wiens von ca. 230.000 EinwohnerInnen im Jahr 1800 auf ca. 700.000 angestiegen. Mit der Industrialisierung wurde ein weiterer Anstieg der Bevölkerung erwartet und die Stadt suchte geeignete Flächen, um die Nachfrage nach Wohn-, Gewerbe- und Industriestandorten zu decken. Die Donauauen wurden zum begehrten Stadtentwicklungsraum und die seit dem Extremhochwasser 1830 neuerlich und intensiv diskutierte Wiener Donauregulierung erhielt ein weiteres Ziel. Standen bis zur Mitte des 19. Jahrhunderts vor allem die Verbesserung der Schifffahrt und die Errichtung einer stabilen Brücke über die Donau im Vordergrund, so sollten nun auch hochwasserfreie Bauzonen geschaffen werden. Bereits während der Großen Wiener Donauregulierung zwischen 1870 und 1875 entstanden Flächenwidmungspläne, die die Donauauen einbezogen. Jener von Heinrich Grave aus dem Jahr 1874 berücksichtigte z. B. den Norden der Insel zwischen dem Donaukanal und dem künftigen Donauhauptarm (Bezirk Brigittenau) und dessen rechtes, stadtnahes Ufer. Grave bezog in seinem Plan über „Wien und Vororte mit Darstellung der Donau und ihrer Auen vor und nach der Regulierung" aber auch einige Bereiche auf dem linken Donauufer ein, vor allem entlang der zu dieser Zeit bereits bestehenden Nordbahntrasse. Nach dem Abschluss der Donauregulierung griff die Stadt zunehmend in die Donauauen aus, wenngleich das Ausmaß durch die Weltwirtschaftskrise zunächst gedämpft war. Bis kurz vor dem Ersten Weltkrieg waren jedoch zwischen Donaukanal und neuem Donauhauptarm vor allem um den Nordbahnhof sowie um den 1874 eröffneten Bahnhof der Nordwestbahn ausgedehnte Siedlungsflächen entstanden. Der Prater verblieb weiterhin als Grünzone und tauchte als solche auch in den Flächenwidmungs- plänen auf (s. Abb. 5; Haidvogl et al. 2013).

\section{Schlussfolgerungen}

Eine langfristige Analyse der Landnutzungsänderung und Besiedelung von Flussauen und technischem Hochwasserschutz zeigt die komplexen $\mathrm{Zu}-$ sammenhänge und Wechselwirkungen (s. dazu auch Sivapalan et al. 2012). Auen waren in Österreich begehrte und zunehmend genutzte Siedlungsund Wirtschaftsräume. Historische Entscheidungen schränken heute das Entwicklungspotenzial von Flussauen ein und setzten eine Risikospirale in Gang, in der zunehmend ansteigende Schadenspotenziale derzeit und auch künftig vor Überschwemmungen geschützt werden müssen. Aktuell stellen die vorhandenen Nutzungen sowie die dadurch verursachten ökologischen Folgen (Verlust von Auwäldern, Augewässern, mangelnde Vernetzung mit dem Fluss, häufig eingeschränkte Funktionsfähigkeit noch verbliebener Auenstandorte) das Gewässermanagement vor große Herausforderungen. Diese können nur mithilfe von einzugsgebietsbezogener Planung, der Integration von vielfältigen Anforderungen inklusive der Ökologie und der Berücksichtigung der Raumplanung als Steuerungsinstrument gelöst werden.

Funding Open access funding provided by University of Natural Resources and Life Sciences Vienna (BOKU).

Open Access Dieser Artikel wird unter der Creative Commons Namensnennung 4.0 International Lizenz (http:// creativecommons.org/licenses/by/4 . 0/deed.de) veröffentlicht, welche die Nutzung, Vervielfältigung, Bearbeitung, Verbreitung und Wiedergabe in jeglichem Medium und Format erlaubt, sofern Sie den/die ursprünglichen $\mathrm{Au}$ tor(en) und die Quelle ordnungsgemäß nennen, einen Link zur Creative Commons Lizenz beifügen und angeben, $o b$ Änderungen vorgenommen wurden. 
Bundesministerium für Land- und Forstwirtschaft, Umwelt und Wasserwirtschaft (2005): Flood Risk - Analyse der Hochwasserereignisse vom August 2002. Wien

Deiss, F. (1963): Generelles Projekt für den Hochwasserschutz der Stadt St. Pölten. Technischer Bericht. Original im Amt der Niederösterreichischen Landesregierung, Gruppe Wasser, Abteilung Wasserbau, St. Pölten

Eberstaller, J., Haidvogl, G., Seebacher, F., Pinka, P., Gabriel, H., Fraiß, B., Kusebauch, G. (2004): Raumordnung und Hochwasserschutz am Beispiel der Traisen - Siedlungsentwicklung und Schadensanalyse. Projekt im Rahmen des Forschungsprogramms „Floodrisk“. Projektbericht, Amt der Niederösterreichischen Landesregierung, Gruppe Wasser, St. Pölten Eberstaller, J., Schmalfuß, R., EberstallerFleischanderl, D., Gabriel, H., Kaufmann, T., Wimmer, H., Jungwirth, M. (2018): LIFE+ Traisen: Der neue Fluss - die Umsetzung. Österreichische Wasser- und Abfallwirtschaft. https:// doi.org/10.1007/s00506-018-0474-9

Fraiß, B. (2004): Siedlungsentwicklung im potentiellen HQ100- und HQ300-Überflutungsraum der Traisen und deren flussmorphologische Auswirkungen. Diplomarbeit, Universität für Bodenkultur Wien

Gutkas, K. (1982): Stadt St. Pölten mit Markt Pottenbrunn. Hoffmann A. (Hrsg): Österreichisches Städtebuch. Österreichische Akademie der Wissenschaften, Wien, S. 35-59

Haidvogl, G. (2010): Verschwundene Fische und trockene Auen. Wie Regulierung und Kraftwerksbau das Ökosystem Donau im Machland verändert haben. In: Winiwarter V, Schmid M (Hrsg.) Umwelt Donau: Eine andere Geschichte. Katalog zur Ausstellung des Niederösterreichischen Landesarchivs im ehemaligen Pfarrhof Ardagger Markt, 5. Mai-7. Nov. 2010. NÖ Institut für Landeskunde, St. Pölten, S 118-135

Haidvogl, G., Hohensinner, S., Preis, S. (2011): Rekonstruktion historischer Flusslandschaften als Grundlage im Gewässermanagement - Potentiale und Limits. Österreichische Wasser- und Abfallwirtschaft 63: 174-182
Haidvogl, G., Horvath, M., Gierlinger, S., Hohensinner, S., Sonnlechner, Ch. (2013): Urban land for a growing city at the banks of a moving river: Vienna's spread into the Danube floodplains from the late 17th century until 1912. Water History 5: 195-217

Haidvogl, G. (2008): Von der Flusslandschaft zum Fließgewässer. Die Entwicklung ausgewählter österreichischer Flüsse im 19. und 20. Jahrhundert mit besonderer Berücksichtigung der Kolonisierung des Überflutungsraums. Dissertation, Universität Wien

Hein, T., Lazowski, W., Muhar, S., Schwarz, U., Weigelhofer, G. (2014): Die Bedeutung von Augewässern am Beispiel der Donau-Auen bei Wien. Denisia33, Neue Serie 163: 167-174

Herrmann, A. (1930): Geschichte der Stadt St. Pölten. Sommer, St. Pölten

Höbinger, W. (1970): Wasserwirtschaft im Traisental. Bundesministerium für Land- und Forstwirtschaft (Hrsg): 50 Jahre Traisen - Wasserverband. Wien, S 12-19

Hohensinner, S., Lager, B., Sonnlechner, C., Haidvogl, G., Gierlinger, S., Schmid, M., Krausmann, F., Winiwarter, V. (2013): Changes in water and land: the reconstructed Viennese riverscape from 1500 to the present. Water History 5: 145-172

Internationale Regierungskommission Alpenrhein \& Internationale Rheinregulierung (2005): Entwicklungskonzept Alpenrhein: $\mathrm{Zu}$ kunft Alpenrhein - Kurzbericht. Thusis

Kaufmann, T., Jungwirth, M., Haidvogl, G., Frik, G., Schmalfuß, R., Eberstaller, J. (2018): LIFE+ Traisen: Der lange Weg zum neuen Fluss. Österreichische Wasser- und Abfallwirtschaft. https:// doi.org/10.1007/s00506-018-0481-9

Landesausschuss des Erzherzogtums Österreich unter der Enns (1914): Die Regulierung der Traisen von Göblasbruck bis zur Donau. Wien

Lexer, W., Linser, S. (2005): Nichtnachhaltige Trends in Österreich: Qualitative Lebensraumveränderung durch Flächenverbrauch. Umweltbundesamt Lebensministerium und Forum Nachhaltiges Österreich. Wien
Muhar, S., Poppe, M., Egger, G., Schmutz, S., Melcher, A. (2004): Flusslandschaften Österreichs. Ausweisung von Flusslandschaften anhand des Naturraums, der Fischfauna und der Auenvegetation. Bundesministerium für Wissenschaft, Bildung und Kultur, Wien

Niederösterreichisches Landesbauamt (1886): Bericht über die Regulierung der Traisen und deren Zuflüsse. Das Flussgebiet der Traisen. Handschriftliches Originalmanuskript im Amt der Niederösterreichischen Landesregierung, Gruppe Wasser, Abteilung Wasserbau, St. Pölten Poppe, M., Muhar, S., Egger, G., Schmutz, S. (2003): Status quo der österreichischen Flusslandschaften: Erfassung und Bilanzierung der Eingriffe und Nutzungen. Österreichische Wasser- und Abfallwirtschaft 55: 122-128

Ramsauer, B, (1948): Die österreichische Nährflächenreserve - Das 10. Bundesland. Schriftenreihe des österreichischen Wasserwirtschaftsverbands 12: 1-29

Sivapalan, M., Savenije, H., Blöschl, G. (2012): Socio-Hydrology: A New Science of People and Water. Hydrological Processes 26: 1270-1276

Stein, E. (1928): Die Städte Deutschösterreichs 3: St. Pölten. Dt. Kommunalverlag, Berlin

Stokreiter, F. (1988): Die Entwicklung der Stadtplanung in Niederösterreich (von der Mitte des 19. Jahrhunderts bis 1938). Schriftenreihe des Institutes für Städtebau, Raumplanung u. Raumordnung. Technische Universität Wien, 22

Werneck, H. (1965): Beiträge zur Geschichte der Wasserkraftanlagen an der mittleren und unteren Traisen, Fladnitz, Perschling (Mühlen, Hammer, Großgewerke) von 885-1965. Herzogenburg, Horn

Zand, N. (1997): Geschichte der Stadt St. Pölten von 1900-1950 im Wandel der politischen, sozialen und wirtschaftlichen Umbrüche. Dissertation, Universität Wien

Zehndorfer, K. (1970): Die Traisenregulierung. In: Bundesministerium für Land- und Forstwirtschaft (Hrsg): 50 Jahre Traisen-Wasserverband. Wasserwirtschaft im Traisental. Wien, S 25-40 